\title{
Design and Implementation on Gesture Recognition Game Controller
}

\author{
Shoujin Wang ${ }^{1, a^{*}}$, Ziyang Han ${ }^{1, b}$, Song Guo ${ }^{1, c}$ \\ School of Information \& Control engineering, Shenyang Jianzhu University, Shenyang, China \\ a23917240@qq.com, b36075274@qq.com, c79510772@qq.com
}

\begin{abstract}
Keywords: Gesture recognition; Game controller; Motion capture technology; Centroid algorithm
\end{abstract}
\begin{abstract}
It aims at enriching the gaming experience, setting the game free from the mouse and keyboard and converting the ordinary game into somatosensory game. Using motion capture technology, as the motion capture hardware monocular vision sensor, acquire the real-time movement of the moving object by the focus centroid algorithm to achieve the gesture recognition game controller. Experiments show that gesture recognition game controller designed in this paper is simple to operate, has real-time, high preparatory and takes up less system resources.
\end{abstract}

\section{Introduction}

The Purpose and Meaning of Development. Somatosensory game is a new type of electronic games with the body to feel, breaking the mode of operation in the past simply to handle key input to operate through body movements' change $[1,2]$. Somatosensory game allows players to get into the game through bodies, enjoys the new experience of somatosensory interactive with broad market prospects.

Test the Dynamic Focus Capture Technology in human-computer interaction applications feasibility, results, operational efficiency and other indicators. It has a great significance in dynamic focus capture technology correction technology, improvement and expansion of this article.

Designing Ideas. Gesture recognition game controller is an application software that uses action information that camera captured to control the game operation. Among them, the camera captures the action information is implemented by introducing a dynamic focus capture technology package library [3, 4]. It analyses the calculated dynamic focus parameters, trigger the appropriate action when achieving the standard set by the user.

Considering the scope of dynamic focus capture system application and game controller operating requires higher efficiency, the gesture recognition of the software is mainly based on "local target appears," which sets a plurality of partial identification object, can already combines enough target actions to meet the needs of the general game operations $[5,6]$.

\section{Function Design}

According to software requirements, system functions are divided as follows:

(1) Launch. Initializing related code and launch the camera.

(2) Exit. Exit the software and release all system resources.

(3) Set the background. Set the background image of the Dynamic Focus Capture System.

(4) Adjustment of threshold value / sensitivity. Adjust the operation conditions to fine-tune recognition.

(5) Monitoring settings. Set parameters of setting test actions and triggered operations.

(6) Monitoring start (end). The captured process of start (end) motion.

(7) Monitoring pause (resume). The captured process of pause (resume) motion.

(8) About. Introduce software developers' information and version information.

The function and structure of the system is shown in Fig. 1. 


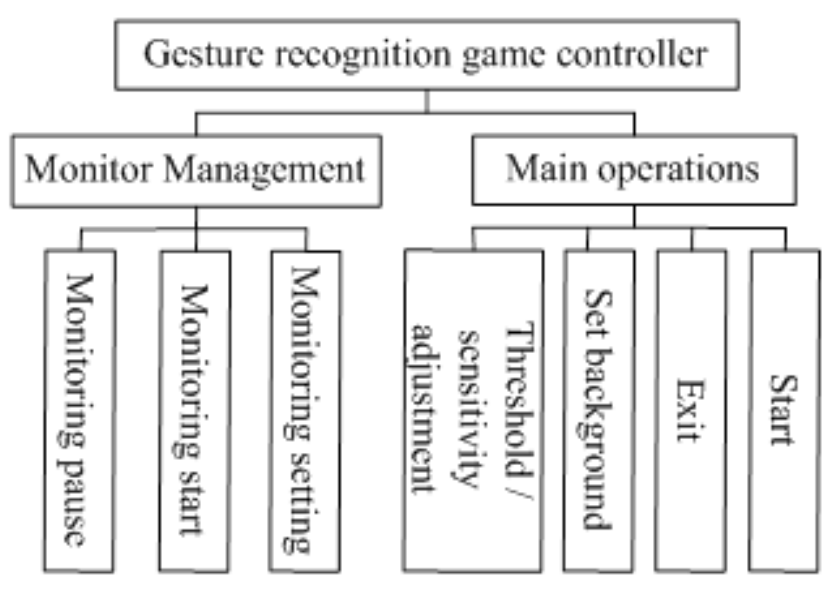

Figure 1. System function structure diagram

\section{Task Procedures}

After entering the main interface of the system, it will load preprocessor code at first. At the moment, the entire system is in standby mode. After receiving the start command, the system will start the video input device, prompt the user to set the background image, and then enter the continuous monitoring process. In the monitoring process, it can monitor the implementation of pause, resume, background updates, adjustment of the threshold, sensitivity adjustment, monitor setting, and monitor ending and other activities at any time.

The task process diagram of the system is shown in Fig. 2.

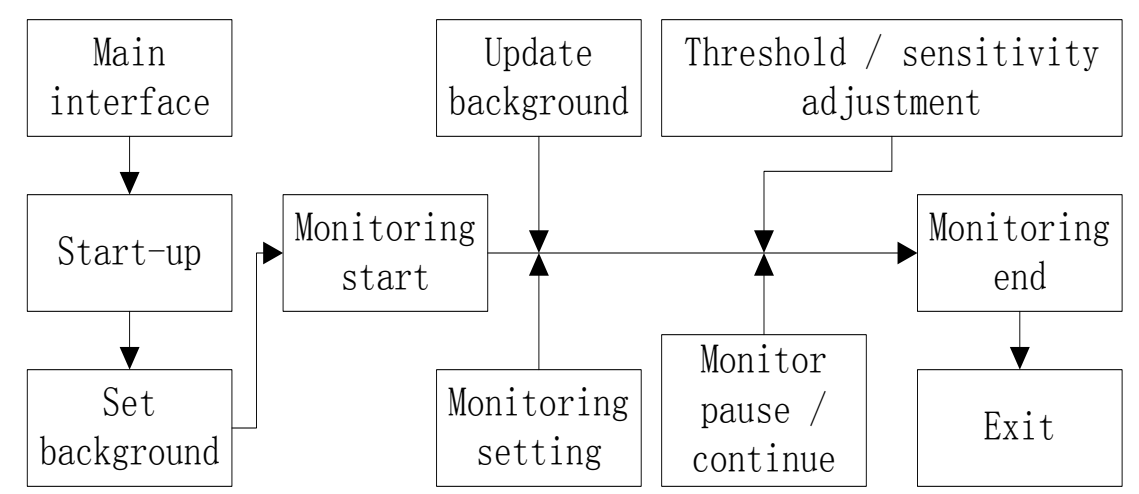

Figure 2. System task progress diagram

\section{Detailed Design}

Main Form Design. The main form is mainly used to provide the entrance of quick access to other sub-functions, by limiting the operability of the control to realize fault-tolerant processing, which means that if the operation $\mathrm{B}$ can be only processed after the operation $\mathrm{A}$, operation $\mathrm{B}$ is inaccessible before operation A being executed.

Start Module Design." Start" function module includes finding and calling camera, updating image data and showing camera windows. "Start" module is a prerequisite for the normal operation of other modules. It is the foundation of the whole system running.

Background Module Setting Design. Update the system background immediately and open the accessibility of "Start Monitoring" function. The process is shown in Fig. 3. 


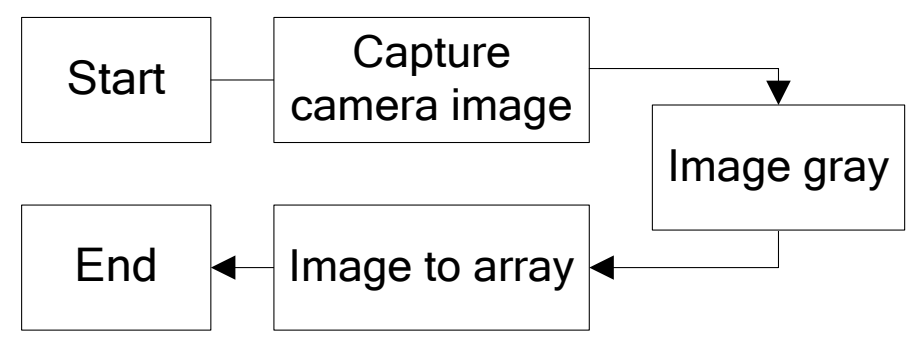

Figure 3. Treatment process

Threshold / Sensitivity Adjustment Module Design. Threshold / sensitivity adjustment module is mainly for adjusting the monitoring parameter is determined so that the entire system can be applied to a variety of external conditions (e.g. illumination, color contrast, range of motion), to monitor and improve the effect[7,8].

Threshold Adjustment. The system uses dynamic capture technology as a core, and the threshold is determined based on the background and the target object (difference) in this technology. When the difference of an image with the background image is greater than a threshold value, it will be judged as a foreground image, and may be determined that the target object image further. When the threshold is too small, it may put a background image due to noise, shadow changes and other factors influence the occurrence of small changes in the image area misjudged the prospects for the region; when the threshold is too high, the target object with no big changed in color may be mistaken for a background image when passing, thereby missing the target determination. Therefore, in order to get the best recognition performance, the threshold value should be scientific and reasonable.

Sensitivity Adjustment. The effects of sensitivity and threshold are similar, are to monitor the effects of fine-tuning. Sensitivity means that only when monitoring over a certain number (area) of the target image will be determined to be a successful recognition. So when the monitor is too sensitive or misinformed, the sensitivity can be increased appropriately; when the monitored target appears lost or not be detected, the sensitivity can be reduced appropriately.

Monitor Setting Module Design. Monitoring module allows users to set capture actions description and trigger related operations directly, can set color filter value, the monitoring area, the trigger button operation of monitored target freely.

The following actions will take setting the target color of action 1 as hand color and trigger button A for and example, to explain the operation flow of monitor set.

Enter the Monitor setting interface. Placed hands in the central range of the camera, click the "Refresh Images Reference" button, then click hand position of the image. Now the text box that is next to "the gray value of mouse clicking point" will be refreshed as the gray value of the hand. Click "Settings" button of action 1 color, set gray value of 174 as the target color of action 1, now the target color box will be refreshed as the corresponding color. Click "target area" button of action 1 to confirm an operation of the monitoring area. Click key selection combo box of action 1 , select "A" key. Click "OK" button to save the settings and return to the previous screen.

\section{Other Main Modules Design.}

1) Start/end monitor module

Introduction: Start/end monitor is used to start and stop the monitoring process related threads. The system identification function only takes effect after starting of the thread after the monitoring.

Pseudo-code of the module is implemented as follows:

Start/end monitor module

\{

If (Monitor has started)

\{

Terminate the associated thread, stop the monitoring process

Settings button prompt text as "Start Monitoring" 


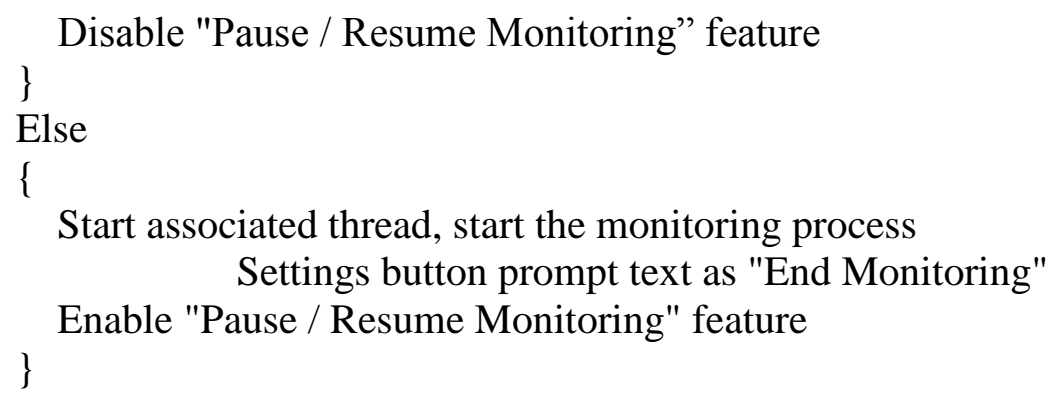

2) Pause/ resume monitor module

Introduction: Pause / resume monitor module is used to suspend and resume the monitoring process related threads. Monitoring should be in the suspended state to adjust the parameters and background updates.

Pseudo-code of the module is implemented as follows:

Pause/resume monitor module

\{

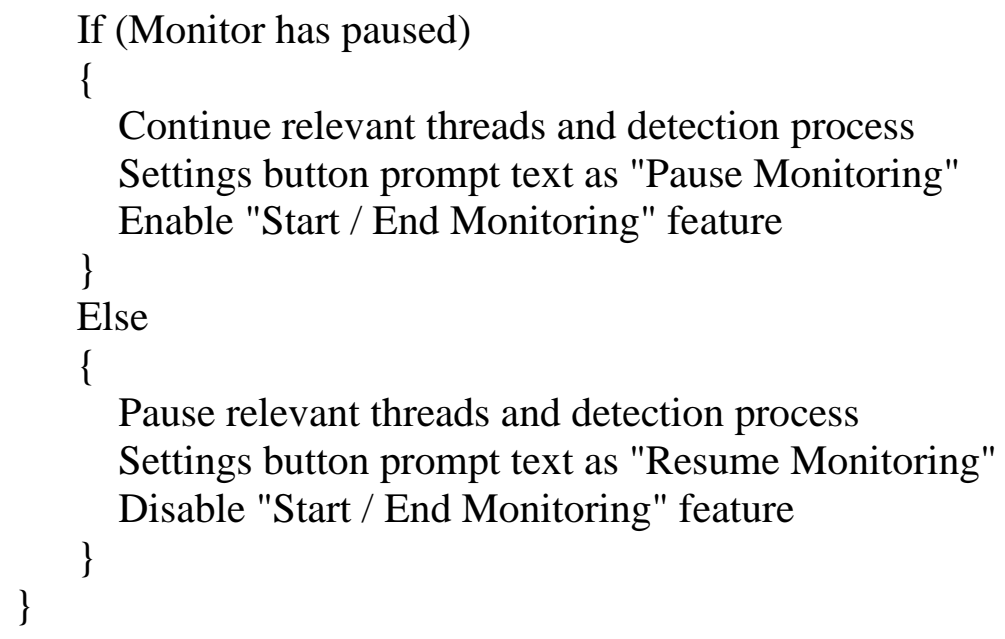

\section{The Software Using Effect}

Gesture recognition game controller is the application software which is based on the player gestures as drivers to operate games. In this test, we realized the functions of operating the left and right baffles of 3D Pinball game.

During the test, the system threshold value is set as 40 , the sensitivity is set as 20 and the image processing size is $320 * 240$.

3D Pinball is a computer game with keyboard operation included in the Windows operating system. The game uses the left and right bezel to hit the pinball, make the pinball hitting the target position to get score. During the test, we set the pressed key of left bezel button as $\mathrm{Z}$ and right bezel buttons as $\mathrm{M}$ of 3D Pinball game.

After the starting software monitor, the system begins gesture identification process. When the user lifts his left hand, the left shutter of 3D Pinball is lifted. When the user puts down the left hand, the left shutter 3D Pinball puts down immediately. When the user lifts his right hand, right-hand flap of 3D Pinball is lifted; when the user puts down the right hand, the right bezel 3D Pinball puts down immediately. Players can hit the ball bezel with gesture control. The software runs properly.

\section{Performance Analyses}

Performance Requirements. When the user doing gesture operations, it should be simple and convenient, have no too high demands of the accuracy of user action. For the immediate action target games, such as racing, action, sports games, real-time directly affect the operation of the reaction to the game experience. For the general users, the latency operation requirements of the 
reactor is less than $50 \mathrm{~ms}$, when the latency operation is greater than $50 \mathrm{~ms}$, users will feel the stuck apparently which may cause bad gaming experience. After the user makes a valid action, the system should be able to judge to be effective action in accuracy and react; when the user does not make an effective operation, the system should not trigger the reaction. The accuracy of the software is the accuracy of the correct recognition of users' gesture.

Accuracy Analysis. After testing, the various parameter settings and the accuracy of actions identified under external environment are shown as Table 1.

Table 1 The accuracy of gesture recognition

\begin{tabular}{|l|l|l|l|}
\hline Threshold & Sensitivity & External environment & $\begin{array}{l}\text { Recognition rate } \\
(20 \text { target gestures })\end{array}$ \\
\hline 50 & 50 & Normal & $95 \%$ \\
\hline 10 & 50 & Dim & $90 \%$ \\
\hline 10 & 50 & Normal & $95 \%$ \\
\hline 50 & 50 & Big change & $95 \%$ \\
\hline 50 & 2 & Normal & $95 \%$ \\
\hline 50 & 2 & Dim & $95 \%$ \\
\hline 50 & 50 & Target color and background are similar & $75 \%$ \\
\hline
\end{tabular}

By adjusting the threshold and sensitivity under normal external environment, it can achieve high recognition accuracy (95\%). Normal external conditions are: relatively bright light conditions, small background disturbance, the gesture recognition target and big the background color difference.

\section{Conclusions}

After analyzing the four aspects as the degree of operation difficulty, real-time, accuracy and resource consumption, the software performs well, achieving the expectations of easy to use for users, quick system response, accurate gesture recognition, low configuration requirements. The software has a good performance.

\section{References}

[1] Xinchen Liu, Huiyuan $\mathrm{Fu}$ and Huadong Ma. Real-time tracking and gesture recognition gesture-based RGB-D camera [D] Beijing: Beijing Youdian University, 2014: 13 14

[2] Yaping Gao. Study based on single camera gesture recognition method [D]. Xiamen: Xiamen University, 2014: 34 35

[3] Fei Liu. Human action recognition based on Kinect skeleton information [D]. Shanghai: Shanghai Donghua University, 2014: 25 27

[4] Min Lin, Bin Li, Qiao-Hong Liu. Identification of Eye Movements from Non-front--al Face Images for Eye-controlled Systems[J]International Journal of Automation \& C-omputing,2014,issue No.5: 29 30

[5] Wei Xiaopeng, Liu Rui, Zhang Qiang. Review of techniques for motion capture data processing[J].Computer Aided Drafting, Design and Manufacturing,2012, issue No.1: 5 7

[6] JIANG Lei, YI Han-fei. A Hand Gesture Recognition Method Based on SVM [J]. Computer Aided Drafting, Design and Manufacturing, 2010, 20(1):85-91.

[7] Xiaopeng Wei, Boxiang Xiao, and Qiang Zhang. A Retrieval Method for Human Mocap Data Based on Biomimetic Pattern Recognition [J]. ComSIS, 2010, 7(1): Special Issue 99-109. 
[8] D.M. Zhu, Z.Q. Wang. Extraction of Keyframe from Motion Capture Data Based on Motion Sequence Segmentation [J]. Journal of Computer-Aided Design \& Computer Graphics, 2008, 20(6): 787-792. 